\title{
An Ostrowski like inequality for convex functions and applications
}

\author{
Sever S. DRAGOMIR \\ School of Computer Science and Mathematics \\ Victoria University of Technology \\ PO Box 14428, Melbourne City MC \\ Victoria 8001, Australia. \\ sever@matilda.vu.edu.au
}

Recibido: 23 de Julio de 2002

Aceptado: 17 de Febrero de 2003

\section{ABSTRACT}

In this paper we point out an Ostrowski type inequality for convex functions which complement in a sense the recent results for functions of bounded variation and absolutely continuous functions. Applications in connection with the Hermite-Hadamard inequality are also considered.

2000 Mathematics Subject Classification: Primary 26D15, 26D10.

Key words: Ostrowski type inequalities, Convex functions, Hermite-Hadamard type inequalities

\section{Introduction}

In 1938, A. Ostrowski [9] proved the following integral inequality

$$
\left|f(x)-\frac{1}{b-a} \int_{a}^{b} f(t) d t\right| \leq\left[\frac{1}{4}+\left(\frac{x-\frac{a+b}{2}}{b-a}\right)^{2}\right](b-a)\left\|f^{\prime}\right\|_{\infty}
$$

provided $f$ is differentiable and $\left\|f^{\prime}\right\|_{\infty}=\sup _{t \in(a, b)}\left|f^{\prime}(t)\right|<\infty$.

The constant $\frac{1}{4}$ is sharp in the sense that it cannot be replaced by a smaller constant.

In the last 5 years, many authors have concentrated their efforts in generalising (1.1) and have applied the obtained results in different fields, including Numerical Integration, Probability Theory and Statistics, Information Theory, etc. For a comprehensive approach in the field, see the recent book [5] where many other references may be found. 
One direction of generalising (1.1) was pointed out by the author in [2] - [4]. Let us recall here a couple of the main results obtained in the above papers.

Theorem 1. Let $I_{k}: a=x_{0}<x_{1}<\cdots<x_{k-1}<x_{k}=b$ be a division of the interval $[a, b]$ and $\alpha_{i}(i=0, \ldots, k+1)$ be $k+2$ points such that $\alpha_{0}=a, \alpha_{i} \in\left[x_{i-1}, x_{i}\right]$ $(i=1, \ldots, k)$ and $\alpha_{k+1}=b$. If $f:[a, b] \rightarrow \mathbb{R}$ is of bounded variation on $[a, b]$, then we have the inequality:

$$
\begin{aligned}
& \left|\int_{a}^{b} f(x) d x-\sum_{i=0}^{k}\left(\alpha_{i+1}-\alpha_{i}\right) f\left(x_{i}\right)\right| \\
\leq & {\left[\frac{1}{2} \nu(h)+\max \left\{\left|\alpha_{i+1}-\frac{x_{i}+x_{i+1}}{2}\right|, i=0, \ldots, k-1\right\}\right] \bigvee_{a}^{b}(f), }
\end{aligned}
$$

where $\nu(h):=\max \left\{h_{i} \mid i=0, \ldots, k-1\right\}, h_{i}:=x_{i+1}-x_{i}(i=0, \ldots, k-1)$ and $\bigvee_{a}^{b}(f)$ is the total variation of $f$ on $[a, b]$.

The constant $\frac{1}{2}$ is sharp in the sense that it cannot be replaced by a smaller constant.

If one would assume more for the function $f$, for example, absolute continuity, then the following result holds.

Theorem 2. Under the assumptions of Theorem 1 for $I_{k}$ and $\alpha_{i}(i=0, \ldots, k+1)$ and if $f:[a, b] \rightarrow \mathbb{R}$ is absolutely continuous on $[a, b]$, then

$$
\leq \begin{cases}{\left[\int_{a}^{b} f(x) d x-\sum_{i=0}^{k}\left(\alpha_{i+1}-\alpha_{i}\right) f\left(x_{i}\right) \mid\right.} & \text { if } f^{\prime} \in L_{\infty}[a, b] ; \\ \frac{1}{(q+1)^{\frac{1}{q}}}\left[\sum_{i=0}^{k-1}\left[\left(\alpha_{i+1}-x_{i}\right)^{q+1}+\sum_{i=0}^{k-1}\left(\alpha_{i+1}-\frac{x_{i}+x_{i+1}}{2}\right)^{2}\right]\left\|f^{\prime}\right\|_{\infty}\right. & \text { if } f^{\prime} \in L_{p}[a, b], \\ \left.\left.\left[\frac{1}{2} \nu(h)+\max \left\{\left|\alpha_{i+1}-\frac{x_{i}+x_{i+1}}{2}\right|, i=0, \ldots, k-1\right\}\right] \| \alpha^{\prime}\right)^{q+1}\right] \|_{1}, & p>1, \frac{1}{p}+\frac{1}{q}=1 ;\end{cases}
$$

where $\|\cdot\|_{p}(p \in[1, \infty])$ are the Lebesgue norms, i.e.,

$$
\begin{aligned}
\|h\|_{\infty}: & =e s s \sup _{t \in[a, b]}|h(t)| \\
\|h\|_{p}: & =\left(\int_{a}^{b}|h(t)|^{p} d t\right)^{\frac{1}{p}}, \quad p \in[1, \infty) .
\end{aligned}
$$


The constants $\frac{1}{4}, \frac{1}{(q+1)^{\frac{1}{q}}}$ and $\frac{1}{2}$ are best in the sense mentioned above.

In this paper, the case of convex functions $f:[a, b] \rightarrow \mathbb{R}$ is examined. Some particular cases in connection with the well known Hermite-Hadamard inequality for convex functions are also considered.

\section{The Results}

The following result holds.

Theorem 3. Let $I_{k}: a=x_{0}<x_{1}<\cdots<x_{k-1}<x_{k}=b$ be a division of the interval $[a, b]$ and $\alpha_{i}(i=0, \ldots, k+1)$ be $k+2$ points such that $\alpha_{0}=a, \alpha_{i} \in\left[x_{i-1}, x_{i}\right]$ $(i=1, \ldots, k)$ and $\alpha_{k+1}=b$. If $f:[a, b] \rightarrow \mathbb{R}$ is a convex function on $[a, b]$, then we have the inequality:

$$
\begin{aligned}
& \frac{1}{2} \sum_{i=0}^{k-1}\left[\left(x_{i+1}-\alpha_{i+1}\right)^{2} f_{+}^{\prime}\left(\alpha_{i+1}\right)-\left(\alpha_{i+1}-x_{i}\right)^{2} f_{-}^{\prime}\left(\alpha_{i+1}\right)\right] \\
\leq & \sum_{i=0}^{k}\left(\alpha_{i+1}-\alpha_{i}\right) f\left(x_{i+1}\right)-\int_{a}^{b} f(t) d t \\
\leq & \frac{1}{2} \sum_{i=0}^{k-1}\left[\left(x_{i+1}-\alpha_{i+1}\right)^{2} f_{-}^{\prime}\left(x_{i+1}\right)-\left(\alpha_{i+1}-x_{i}\right)^{2} f_{+}^{\prime}\left(x_{i}\right)\right] .
\end{aligned}
$$

The constant $\frac{1}{2}$ is sharp in both inequalities.

Proof. Using the integration by parts formula, we may prove the equality (see for example [3]):

$$
\sum_{i=0}^{k}\left(\alpha_{i+1}-\alpha_{i}\right) f\left(x_{i+1}\right)-\int_{a}^{b} f(t) d t=\sum_{i=0}^{k-1} \int_{x_{i}}^{x_{i+1}}\left(t-\alpha_{i+1}\right) f^{\prime}(t) d t
$$

for any locally absolutely continuous function $f:(a, b) \rightarrow \mathbb{R}$.

Since $f$ is convex, then it is locally Lipschitzian on $(a, b)$ and thus the above equality holds. Also, we have

$$
f_{+}^{\prime}\left(x_{i}\right) \leq f^{\prime}(t) \leq f_{-}^{\prime}\left(\alpha_{i+1}\right) \text { for a.e. } t \in\left[x_{i}, \alpha_{i+1}\right]
$$

and

$$
f_{+}^{\prime}\left(\alpha_{i+1}\right) \leq f^{\prime}(t) \leq f_{-}^{\prime}\left(x_{i+1}\right) \text { for a.e. } t \in\left[\alpha_{i+1}, x_{i+1}\right] .
$$

Using (2.3) and (2.4), we may write that

$$
\begin{aligned}
f_{-}^{\prime}\left(\alpha_{i+1}\right) \int_{x_{i}}^{\alpha_{i+1}}\left(t-\alpha_{i+1}\right) d t & \leq \int_{x_{i}}^{\alpha_{i+1}} f^{\prime}(t)\left(t-\alpha_{i+1}\right) d t \\
& \leq f_{+}^{\prime}\left(x_{i}\right) \int_{x_{i}}^{\alpha_{i+1}}\left(t-\alpha_{i+1}\right) d t
\end{aligned}
$$


and

$$
\begin{aligned}
f_{+}^{\prime}\left(\alpha_{i+1}\right) \int_{\alpha_{i+1}}^{x_{i+1}}\left(t-\alpha_{i+1}\right) d t & \leq \int_{\alpha_{i+1}}^{x_{i+1}} f^{\prime}(t)\left(t-\alpha_{i+1}\right) d t \\
& \leq f_{-}^{\prime}\left(x_{i+1}\right) \int_{\alpha_{i+1}}^{x_{i+1}}\left(t-\alpha_{i+1}\right) d t .
\end{aligned}
$$

Adding (2.5) and (2.6) and taking into account that

$$
\int_{x_{i}}^{\alpha_{i+1}}\left(t-\alpha_{i+1}\right) d t=-\frac{1}{2}\left(\alpha_{i+1}-x_{i}\right)^{2}
$$

and

$$
\int_{\alpha_{i+1}}^{x_{i+1}}\left(t-\alpha_{i+1}\right) d t=\frac{1}{2}\left(x_{i+1}-\alpha_{i+1}\right)^{2}
$$

we get

$$
\begin{aligned}
& \frac{1}{2}\left[\left(x_{i+1}-\alpha_{i+1}\right)^{2} f_{+}^{\prime}\left(\alpha_{i+1}\right)-\left(\alpha_{i+1}-x_{i}\right)^{2} f_{-}^{\prime}\left(\alpha_{i+1}\right)\right] \\
\leq & \int_{x_{i}}^{x_{i+1}}\left(t-\alpha_{i+1}\right) f^{\prime}(t) d t \\
\leq & \frac{1}{2}\left[\left(x_{i+1}-\alpha_{i+1}\right)^{2} f_{-}^{\prime}\left(x_{i+1}\right)-\left(\alpha_{i+1}-x_{i}\right)^{2} f_{+}^{\prime}\left(x_{i}\right)\right]
\end{aligned}
$$

for any $i=0, \ldots, k-1$.

If we sum (2.7) over $i$ from 0 to $k-1$ and use the identity (2.2), we deduce the desired result (2.1).

The sharpness will be proved in what follows for a particular case.

It is natural to consider the following particular case.

Corollary 1. Let $L_{k}$ and $f$ be as in the above theorem. Then we have the inequality

$$
\begin{aligned}
0 \leq & \frac{1}{8} \sum_{i=0}^{k-1}\left[f_{+}^{\prime}\left(\frac{x_{i}+x_{i+1}}{2}\right)-f_{-}^{\prime}\left(\frac{x_{i}+x_{i+1}}{2}\right)\right]\left(x_{i+1}-x_{i}\right)^{2} \\
\leq & \frac{1}{2}\left[\left(x_{1}-a\right) f(a)+\sum_{i=1}^{k-1}\left(x_{i+1}-x_{i-1}\right) f\left(x_{i}\right)+\left(b-x_{k-1}\right) f(b)\right] \\
& -\int_{a}^{b} f(t) d t \\
\leq & \frac{1}{8} \sum_{i=0}^{k-1}\left[f_{-}^{\prime}\left(x_{i+1}\right)-f_{+}^{\prime}\left(x_{i}\right)\right]\left(x_{i+1}-x_{i}\right)^{2} .
\end{aligned}
$$

The constant $\frac{1}{8}$ in both inequalities is sharp. 
The proof follows by the above theorem choosing $\alpha_{i}=\frac{x_{i-1}+x_{i}}{2}, i=1, \ldots, k$ and taking into account that (see also [2])

$$
\begin{aligned}
& \sum_{i=0}^{k}\left(\alpha_{i+1}-\alpha_{i}\right) f\left(x_{i}\right) \\
= & \frac{1}{2}\left[\left(x_{1}-a\right) f(a)+\sum_{i=1}^{k-1}\left(x_{i+1}-x_{i-1}\right) f\left(x_{i}\right)+\left(b-x_{k-1}\right) f(b)\right] .
\end{aligned}
$$

The following corollary for equidistant partitioning also holds.

Corollary 2. Let

$$
I_{k}: x_{i}:=a+(b-a) \cdot \frac{i}{k} \quad(i=0, \ldots, k)
$$

be an equidistant partitioning of $[a, b]$. If $f:[a, b] \rightarrow \mathbb{R}$ is convex on $[a, b]$, then we have the inequalities

$$
\begin{aligned}
0 \leq & \frac{(b-a)^{2}}{8 n^{2}} \sum_{i=0}^{k-1}\left\{f_{+}^{\prime}\left[a+\left(i+\frac{1}{2}\right) \frac{b-a}{n}\right]\right. \\
& \left.-f_{-}^{\prime}\left[a+\left(i+\frac{1}{2}\right) \frac{b-a}{n}\right]\right\} \\
\leq & \frac{1}{k} \cdot \frac{f(a)+f(b)}{2}(b-a) \\
& +\frac{b-a}{k} \sum_{i=1}^{k-1} f\left[\frac{(k-i) a+i b}{k}\right]-\int_{a}^{b} f(t) d t \\
\leq & \frac{(b-a)^{2}}{8 n^{2}} \sum_{i=0}^{k-1}\left\{f_{-}^{\prime}\left[a+(i+1) \cdot \frac{b-a}{n}\right]-f_{+}^{\prime}\left[a+i \cdot \frac{b-a}{n}\right]\right\} .
\end{aligned}
$$

The following particular cases which hold when we assume differentiability conditions may be stated.

Corollary 3. If $\alpha_{i} \in(a, b)$ for $i=1, \ldots, k$ are points of differentiability for $f$, then we have the inequality

$$
\begin{aligned}
& \sum_{i=0}^{k-1}\left(x_{i+1}-x_{i}\right)\left(\frac{x_{i}+x_{i+1}}{2}-\alpha_{i+1}\right) f^{\prime}\left(\alpha_{i+1}\right) \\
\leq & \sum_{i=0}^{k}\left(\alpha_{i+1}-\alpha_{i}\right) f\left(x_{i+1}\right)-\int_{a}^{b} f(t) d t .
\end{aligned}
$$


If we denote by $\nu\left(I_{n}\right):=\max \left\{x_{i+1}-x_{i} \mid i=0, \ldots, k-1\right\}$, then the following corollary also holds.

Corollary 4. If $x_{i}(i=1, \ldots, k-1)$ are points of differentiability for $f$ then

$$
\begin{aligned}
& \frac{1}{2}\left[\left(x_{1}-a\right) f(a)+\sum_{i=0}^{k-1}\left(x_{i+1}-x_{i-1}\right) f\left(x_{i}\right)+\left(b-x_{k-1}\right) f(b)\right]-\int_{a}^{b} f(t) d t \\
\leq & \frac{1}{8}\left[\nu\left(I_{n}\right)\right]^{2}\left[f_{-}^{\prime}(b)-f_{+}^{\prime}(a)\right] .
\end{aligned}
$$

\section{Some Particular Inequalities}

(1) If we choose $x_{0}=a, x_{1}=b, \alpha_{0}=a, \alpha_{1}=x \in(a, b), \alpha_{2}=b$, then from (2.1) we deduce (see also [6])

$$
\begin{aligned}
& \frac{1}{2}\left[(b-x)^{2} f_{+}^{\prime}(x)-(x-a)^{2} f_{-}^{\prime}(x)\right] \\
\leq & (x-a) f(a)+(b-x) f(b)-\int_{a}^{b} f(t) d t \\
\leq & \frac{1}{2}\left[(b-x)^{2} f_{-}^{\prime}(b)-(x-a)^{2} f_{-}^{\prime}(a)\right] .
\end{aligned}
$$

The constant $\frac{1}{2}$ is sharp in both inequalities (see for example [6]).

If $x=\frac{a+b}{2}$, then by (3.1) one deduces (see also [6])

$$
\begin{aligned}
0 & \leq \frac{1}{8}(b-a)^{2}\left[f_{+}^{\prime}\left(\frac{a+b}{2}\right)-f_{-}^{\prime}\left(\frac{a+b}{2}\right)\right] \\
& \leq \frac{f(a)+f(b)}{2} \cdot(b-a)-\int_{a}^{b} f(t) d t \\
& \leq \frac{1}{8}(b-a)^{2}\left[f_{-}^{\prime}(b)-f_{+}^{\prime}(a)\right]
\end{aligned}
$$

and the constant $\frac{1}{8}$ in both inequalities is sharp (see for example [6]).

If one would assume that $x \in(a, b)$ is a point of differentiability, then

$$
(b-a)\left(\frac{a+b}{2}-x\right) f^{\prime}(x) \leq(x-a) f(a)+(b-x) f(b)-\int_{a}^{b} f(t) d t .
$$


(2) If we choose $a=x_{0}<x<x_{2}=b$ and the numbers $\alpha_{0}=a, \alpha \in(a, x], \beta \in[x, b)$ and $\alpha_{3}=b$, then by Theorem 3 , we deduce

$$
\begin{aligned}
& \frac{1}{2}\left[(x-\alpha)^{2} f_{+}^{\prime}(\alpha)-(\alpha-a)^{2} f_{-}^{\prime}(\alpha)+(b-\beta)^{2} f_{+}^{\prime}(\beta)-(\beta-x)^{2} f_{-}^{\prime}(\beta)\right] \\
\leq & (\alpha-a) f(a)+(\beta-\alpha) f(x)+(b-\beta) f(b)-\int_{a}^{b} f(t) d t \\
\leq & \frac{1}{2}\left[(x-\alpha)^{2} f_{-}^{\prime}(x)-(\alpha-a)^{2} f_{+}^{\prime}(a)+(b-\beta)^{2} f_{-}^{\prime}(b)-(\beta-x)^{2} f_{+}^{\prime}(x)\right] .
\end{aligned}
$$

The constant $\frac{1}{2}$ is sharp in both inequalities.

(a) Note that if we let $\alpha \rightarrow a+$ and $\beta \rightarrow b-$, then from (3.4), by taking into account firstly that $(x-\alpha)^{2} f_{+}^{\prime}(a) \leq(x-\alpha)^{2} f_{+}^{\prime}(\alpha)$ and $-(\beta-x)^{2} f_{-}^{\prime}(b) \leq$ $-(\beta-x)^{2} f_{-}^{\prime}(\beta)$, we may deduce the inequality obtained in [7]:

$$
\begin{aligned}
& \frac{1}{2}\left[(b-x)^{2} f_{+}^{\prime}(x)-(x-a)^{2} f_{-}^{\prime}(x)\right] \\
\leq & \int_{a}^{b} f(t) d t-(b-a) f(x) \\
\leq & \frac{1}{2}\left[(\beta-x)^{2} f_{-}^{\prime}(b)+(x-a)^{2} f_{+}^{\prime}(a)\right] .
\end{aligned}
$$

The constant $\frac{1}{2}$ is sharp in both inequalities (see for example [7]).

If in (3.5) we choose $x=\frac{a+b}{2}$, then (see also [7])

$$
\begin{aligned}
0 & \leq \frac{1}{8}(b-a)^{2}\left[f_{+}^{\prime}\left(\frac{a+b}{2}\right)-f_{-}^{\prime}\left(\frac{a+b}{2}\right)\right] \\
& \leq \int_{a}^{b} f(t) d t-(b-a) f\left(\frac{a+b}{2}\right) \\
& \leq \frac{1}{8}(b-a)^{2}\left[f_{-}^{\prime}(b)-f_{+}^{\prime}(a)\right]
\end{aligned}
$$

and the constant $\frac{1}{8}$ is sharp in both inequalities.

We may state now the following result for convex functions improving Hermite-Hadamard integral inequalities. 
Proposition 1. Let $f:[a, b] \rightarrow \mathbb{R}$ be a convex function on $[a, b]$. Then

$$
\begin{aligned}
0 & \leq \frac{1}{8}(b-a)\left[f_{+}^{\prime}\left(\frac{a+b}{2}\right)-f_{-}^{\prime}\left(\frac{a+b}{2}\right)\right] \\
& \leq \frac{1}{b-a} \int_{a}^{b} f(t) d t-f\left(\frac{a+b}{2}\right) \\
& \leq \frac{f(a)+f(b)}{2}-\frac{1}{b-a} \int_{a}^{b} f(t) d t \\
& \leq \frac{1}{8}(b-a)\left[f_{-}^{\prime}(b)-f_{+}^{\prime}(a)\right] .
\end{aligned}
$$

The constant $\frac{1}{8}$ is sharp in both parts.

If one would assume that $x \in(a, b)$ is a differentiability point for $f$, then we have the inequality $[7]$

$$
(b-a)\left(\frac{a+b}{2}-x\right) f^{\prime}(x) \leq \int_{a}^{b} f(t) d t-(b-a) f(x) .
$$

(b) If we choose $\alpha=\frac{a+x}{2}$ and $\beta=\frac{x+b}{2}$, then by (3.4) we have the three point inequality:

$$
\begin{aligned}
0 \leq & \frac{1}{8}\left\{(x-a)^{2}\left[f_{+}^{\prime}\left(\frac{a+x}{2}\right)-f_{-}^{\prime}\left(\frac{a+x}{2}\right)\right]\right. \\
& \left.\quad+(b-x)^{2}\left[f_{+}^{\prime}\left(\frac{x+b}{2}\right)-f_{-}^{\prime}\left(\frac{x+b}{2}\right)\right]\right\} \\
\leq & \frac{1}{2}[(x-a) f(a)+f(x)(b-a)+(b-x) f(b)]-\int_{a}^{b} f(t) d t \\
\leq & \frac{1}{8}\left\{(x-a)^{2}\left[f_{+}^{\prime}(x)-f_{-}^{\prime}(a)\right]+(b-x)^{2}\left[f_{-}^{\prime}(b)-f_{+}^{\prime}(x)\right]\right\}
\end{aligned}
$$

for any $x \in(a, b)$. The constant $\frac{1}{8}$ is sharp in both parts.

If in (3.9) we choose $x=\frac{a+b}{2}$, then we get

$$
\begin{aligned}
0 & \frac{1}{32}(b-a)^{2}\left[f_{+}^{\prime}\left(\frac{3 a+b}{4}\right)-f_{-}^{\prime}\left(\frac{3 a+b}{4}\right)\right. \\
& \left.+f_{+}^{\prime}\left(\frac{a+3 b}{4}\right)-f_{-}^{\prime}\left(\frac{a+3 b}{4}\right)\right] \\
\leq & \frac{1}{2} \cdot\left[\frac{f(a)+f(b)}{2}+f\left(\frac{a+b}{2}\right)\right](b-a)-\int_{a}^{b} f(t) d t \\
\leq & \frac{1}{32}(b-a)^{2}\left[f_{-}^{\prime}(b)-f_{+}^{\prime}\left(\frac{a+b}{2}\right)+f_{-}^{\prime}\left(\frac{a+b}{2}\right)-f_{+}^{\prime}(a)\right]
\end{aligned}
$$


If one would assume that $f$ is differentiable in $\frac{a+b}{2}$, then we get the following reverse of Bullen's inequality

$$
\begin{aligned}
0 & \leq \frac{1}{2} \cdot\left[\frac{f(a)+f(b)}{2}+f\left(\frac{a+b}{2}\right)\right](b-a)-\int_{a}^{b} f(t) d t \\
& \leq \frac{1}{32}(b-a)^{2}\left[f_{-}^{\prime}(b)-f_{+}^{\prime}(a)\right] .
\end{aligned}
$$

The constant $\frac{1}{32}$ is sharp.

(c) Now, if we choose $\alpha=\frac{5 a+b}{6}, \beta=\frac{a+5 b}{6}$ and $x \in\left[\frac{5 a+b}{6}, \frac{a+5 b}{6}\right]$ in (3.4), then we have the inequalities

$$
\begin{aligned}
& \frac{1}{2}\left[\left(x-\frac{5 a+b}{6}\right)^{2} f_{+}^{\prime}\left(\frac{5 a+b}{6}\right)-\frac{(b-a)^{2}}{36} f_{-}^{\prime}\left(\frac{5 a+b}{6}\right)\right. \\
& \left.+\frac{(b-a)^{2}}{36} f_{+}^{\prime}\left(\frac{a+5 b}{6}\right)-\left(\frac{a+5 b}{6}-x\right)^{2} f_{-}^{\prime}\left(\frac{a+5 b}{6}\right)\right] \\
\leq & \frac{b-a}{3}\left[\frac{f(a)+f(b)}{2}+2 f(x)\right]-\int_{a}^{b} f(t) d t \\
\leq & \frac{1}{2}\left[\left(x-\frac{5 a+b}{6}\right)^{2} f_{-}^{\prime}(x)-\frac{(b-a)^{2}}{36} f_{+}^{\prime}(a)\right. \\
& \left.+\frac{(b-a)^{2}}{36} f_{-}^{\prime}(b)-\left(\frac{a+5 b}{6}-x\right)^{2} f_{+}^{\prime}(x)\right] .
\end{aligned}
$$

If in (3.12) we choose $x=\frac{a+b}{2}$, then we get the Simpson's inequality

$$
\begin{aligned}
& \frac{1}{18}(b-a)^{2}\left[f_{+}^{\prime}\left(\frac{5 a+b}{6}\right)-\frac{1}{4} f_{-}^{\prime}\left(\frac{5 a+b}{6}\right)\right. \\
& \left.+\frac{1}{4} f_{+}^{\prime}\left(\frac{a+5 b}{6}\right)-f_{-}^{\prime}\left(\frac{a+5 b}{6}\right)\right] \\
\leq & \frac{b-a}{3}\left[\frac{f(a)+f(b)}{2}+2 f\left(\frac{a+b}{2}\right)\right]-\int_{a}^{b} f(t) d t \\
\leq & \frac{1}{18}(b-a)^{2}\left[f_{-}^{\prime}\left(\frac{a+b}{2}\right)-\frac{1}{4} f_{+}^{\prime}(a)+\frac{1}{4} f_{-}^{\prime}(b)-f_{+}^{\prime}\left(\frac{a+b}{2}\right)\right] .
\end{aligned}
$$


If the function is differentiable on $(a, b)$, then we get

$$
\begin{aligned}
& -\frac{1}{24}(b-a)^{2}\left[f^{\prime}\left(\frac{a+5 b}{6}\right)-f^{\prime}\left(\frac{5 a+b}{6}\right)\right] \\
\leq & \frac{b-a}{3}\left[\frac{f(a)+f(b)}{2}+2 f\left(\frac{a+b}{2}\right)\right]-\int_{a}^{b} f(t) d t \\
\leq & \frac{1}{72}(b-a)^{2}\left[f_{-}^{\prime}(b)-f_{+}^{\prime}(a)\right] .
\end{aligned}
$$

\section{References}

[1] G. Anastassiou, Ostrowski type inequalities, Proc. Amer. Math. Soc., 123(12) (1995), 3775-3781.

[2] S.S. Dragomir, The Ostrowski integral inequality for mappings of bounded variation, Bull. Austral. Math. Soc., 60 (1999), 495-508.

[3] S.S. Dragomir, A generalisation of Ostrowski integral inequality for mappings whose derivatives belong to $L_{p}[a, b], 1<p<\infty$ and applications in numerical integration, $J$. Math. Anal. Appl., 225 (2001), 605-626.

[4] S.S. Dragomir, A generalisation of Ostrowski integral inequality for mappings whose derivatives belong to $L_{1}[a, b]$, and applications in numerical integration, J. Computational Analysis and Appl., 3(4) (2001), 343-360.

[5] S.S. Dragomir and Th. M. Rassias (Eds), Ostrowski Type Inequalities and Applications in Numerical Integration, Kluwer Academic Publishers, Dordrecht, 2002.

[6] S.S. Dragomir, A generalised trapezoid type inequality for convex functions, (Preprint) RGMIA Res. Rep. Coll., 5(1) (2002), Article 9. [ONLINE] http://rgmia.vu.edu.au/v5n1.html

[7] S.S. Dragomir, An Ostrowski type inequality for convex functions, (Preprint) RGMIA Res. Rep. Coll., 5(1) (2002), Article 5. [ONLINE] http://rgmia.vu.edu.au/v5n1.html

[8] A.M. Fink, Bounds on the derivation of a function from its averages, Czech. Math. J., 42 (1992), 289-310.

[9] A. Ostrowski, Über die Absolutabweichung einer differentiienbaren Funcktion von ihren Integralwittelwert, Comment. Math. Helv., 10 (1938), 220-227. 